\title{
Engineering of Serine-Deamination pathway, Entner-Doudoroff pathway and pyruvate dehydrogenase complex to improve poly(3-hydroxybutyrate) production in Escherichia coli
}

Yan Zhang ${ }^{1,2,3,5}$, Zhenquan Lin ${ }^{1,2,3,5}$, Qiaojie Liu' ${ }^{1,2,3,5}$, Yifan Li ${ }^{1,2,3,5}$, Zhiwen Wang ${ }^{1,2,3,5}$, Hongwu Ma ${ }^{4}$, Tao Chen ${ }^{1,2,3,5^{*}}$ and Xueming Zhao ${ }^{1,2,3,5}$

\begin{abstract}
Background: Poly(3-hydroxybutyrate) (PHB), a biodegradable bio-plastic, is one of the most common homopolymer of polyhydroxyalkanoates (PHAs). PHB is synthesized by a variety of microorganisms as intracellular carbon and energy storage compounds in response to environmental stresses. Bio-based production of PHB from renewable feedstock is a promising and sustainable alternative to the petroleum-based chemical synthesis of plastics. In this study, a novel strategy was applied to improve the PHB biosynthesis from different carbon sources.

Results: In this research, we have constructed E. coli strains to produce PHB by engineering the Serine-Deamination (SD) pathway, the Entner-Doudoroff (ED) pathway, and the pyruvate dehydrogenase (PDH) complex. Firstly, co-overexpression of sdaA (encodes L-serine deaminase), L-serine biosynthesis genes and pgk (encodes phosphoglycerate kinase) activated the SD Pathway, and the resulting strain SD02 (pBHR68), harboring the PHB biosynthesis genes from Ralstonia eutropha, produced $4.86 \mathrm{~g} / \mathrm{L}$ PHB using glucose as the sole carbon source, representing a 2.34-fold increase compared to the reference strain. In addition, activating the ED pathway together with overexpressing the PDH complex further increased the PHB production to $5.54 \mathrm{~g} / \mathrm{L}$ with content of 81.1\% CDW. The intracellular acetyl-CoA concentration and the $[\mathrm{NADPH}] /\left[\mathrm{NADP}^{+}\right]$ratio were enhanced after the modification of SD pathway, ED pathway and the PDH complex. Meanwhile, these engineering strains also had a significant increase in PHB concentration and content when xylose or glycerol was used as carbon source.

Conclusions: Significant levels of PHB biosynthesis from different kinds of carbon sources can be achieved by engineering the Serine-Deamination pathway, Entner-Doudoroff pathway and pyruvate dehydrogenase complex in $E$. coli JM109 harboring the PHB biosynthesis genes from Ralstonia eutropha. This work demonstrates a novel strategy for improving PHB production in E. coli. The strategy reported here should be useful for the bio-based production of PHB from renewable resources.
\end{abstract}

Keywords: Escherichia coli, poly(3-hydroxybutyrate), L-serine deaminate, Entner-Doudoroff pathway, Pyruvate dehydrogenase complex

\footnotetext{
* Correspondence: chentao@tju.edu.cn

Equal contributors

'Key Laboratory of Systems Bioengineering (Ministry of Education), Tianjin University, Tianjin 300072, People s Republic of China

${ }^{2}$ SynBio Research Platform, Collaborative Innovation Center of Chemical Science and Engineering (Tianjin), School of Chemical Engineering and Technology, Tianjin University, Tianjin 300072, People s Republic of China Full list of author information is available at the end of the article
} 


\section{Background}

Polyhydroxyalkanoates (PHAs) are diverse polyesters synthesized by a variety of microorganisms as intracellular carbon and energy storage compounds in response to environmental stresses [1]. Since PHAs possess thermoplastic or elastomeric properties and are completely biodegradable, PHA bioplastics offer an exciting alternative to petrochemical-derived plastics [2]. Poly(3-hydroxybutyrate) (PHB) is the most wide spread and best-characterized member of PHAs and many different fermentation strategies and recovery methods have been developed for its production as a model polymer [3].

In the majority of native PHB-accumulating species, $\mathrm{PHB}$ is synthesized from acetyl-CoA by a sequence of three enzyme reactions catalyzed by $\beta$-ketothiolase, acetoacetylCoA reductase and PHB synthase, encoded by phaA, phaB and $p h a C$, respectively (Figure 1 ). Recombinant $E$. coli harboring the exogenous PHB synthetic pathway was one of the most frequently used hosts for biopolymer production because of its advantages such as having a wide range of utilizable carbon sources, accumulating of large amounts of polymers with a high level of productivity, high cell density fermentation, and lacking PHA degradation system.

There has been a range of studies to evaluate PHB production in E. coli. Enhancing the availability of the precursor acetyl-CoA or/and cofactor NADPH increased the production of $\mathrm{PHB}$. By overexpressing the fructosebisphosphate aldolase (encoded by $f b a A$ ) or/and triosephosphate isomerase (encoded by tpi) [4], the recombinant E. coli accumulated more PHB than the reference strains due to the increase in acetyl-CoA concentration. By inactivating the phosphoglucose isomerase (encoded by $p g i$ ) gene, more NADPH was produced from pentose phosphate (PP) pathway, and eventually the PHB production was enhanced [5]. Transketolase (encoded by $t k t A$ ) or transaldolase (encoded by talA) was used to improve the metabolism in non-oxidative PP pathway to enhance the production of PHB [6,7]. Overexpressing glucose6-phosphate dehydrogenase (encoded by $z w f$ ) and 6phosphogluconate dehydrogenase (encoded by gnd) in

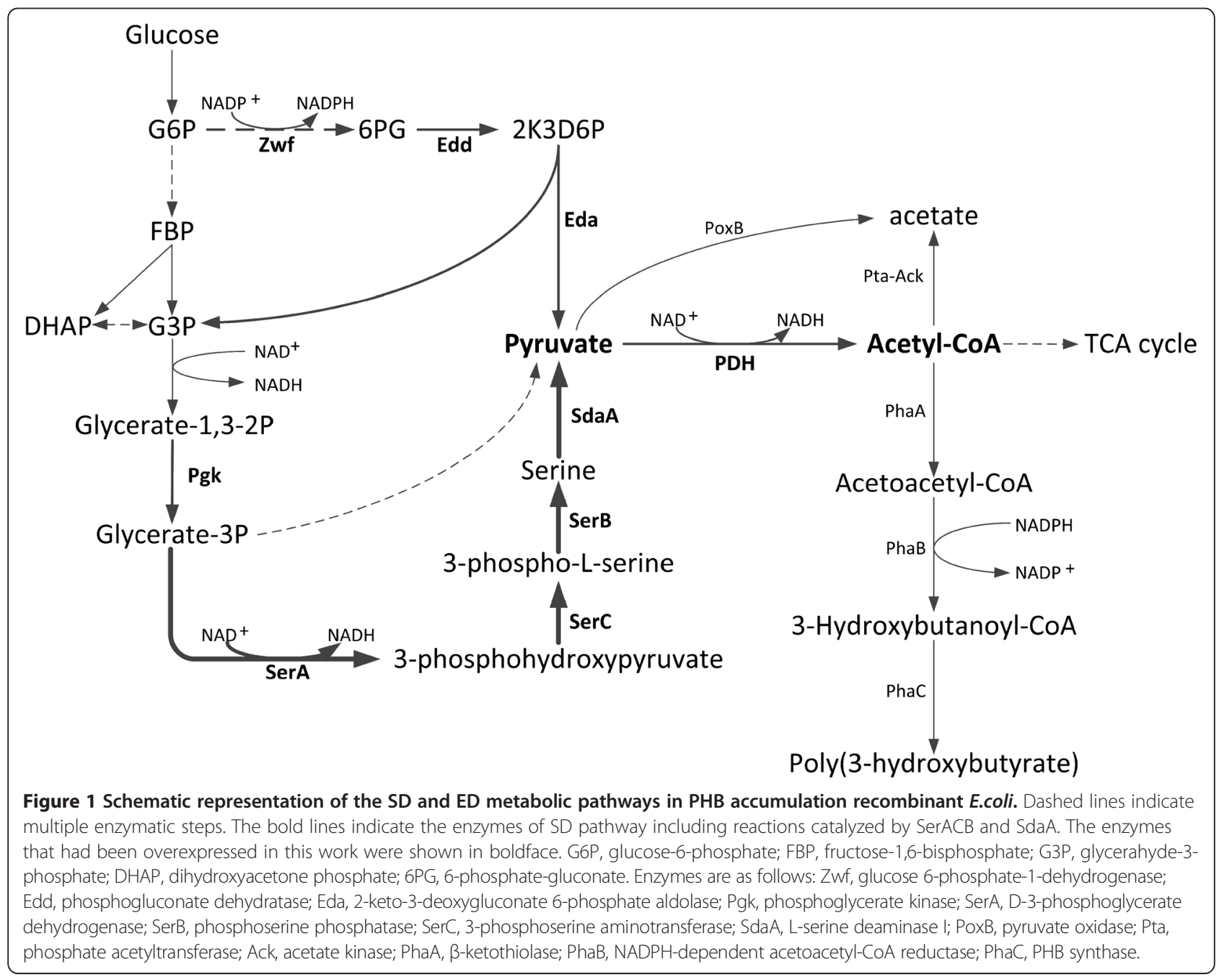


oxidative pentose phosphate pathway increased the PHB production in $E$. coli by increasing the NADPH availability [8]. In addition, to increase product yields, NADPH levels have been manipulated in the past by overexpressing the $\mathrm{NADP}^{+}$-dependent D-glyceraldehyde-3-phosphate dehydrogenase from Streptococcus mutans [9].

In E. coli, acetyl-CoA was derived mostly from pyruvate which is a key intermediate in catabolic and biosynthetic reactions. Most pyruvate was synthesized through the coupled mechanism of glucose transport by the phosphotransferase transport system or the glycolytic pathways including Embden-Meyerhof-Parnas (EMP), Entner-Doudoroff (ED), and PP pathway [10]. E. coli only produced two mole of NADH per mole of glucose through the EMP pathway, and caused the carbon loss through the PP pathway. Compared with these two pathways, ED pathway enables the strain to produce one molecular NADPH, a direct cofactor for the PHB production, without the carbon loss. Moreover, previous research based on proteome analysis revealed that ED pathway plays an important role during PHB production from glucose $[11,12]$. In E. coli, pyruvate also can be synthesized from L-serine by L-serine deaminase [13]. L-serine deaminases (encoded by $s d a A, s d a B$ and $t d c G$ ) [14] catalyze the conversion of L-serine to pyruvate and ammonia, which may be employed to improve the PHB production in E.coli.

In this research, combined engineering of the SerineDeamination Pathway (SD pathway, shown in Figure 1) with ED pathway resulted in enhanced PHB production, but accompanying with pyruvate accumulation. Overexpressing PDH complex eliminated the pyruvate accumulation and led to further improvement of the intracellular acetyl-CoA concentration and PHB production. Finally, the metabolically engineered $E$. coli strain was able to synthesize significant amount of PHB from different carbon sources such as glucose, xylose and glycerol.

\section{Results and discussion}

Overexpressing L-serine deaminase for improved PHB production

Under aerobic or anaerobic conditions, acetyl-CoA was derived mostly from the decarboxylation of pyruvate which respectively catalyzed by the PDH complex or pyruvate-formate lyase in E.coli $[15,16]$. Pyruvate was formed mostly from several glycolysis pathways. Moreover, L-serine derived from D-3-phosphoglycerate can be catalyzed to pyruvate and ammonias by L-serine deaminase. However, L-serine also can be cleaved into glycine and one carbon unit by serine hydroxymethyltransferase (SHMT, encoded by $g l y A$ ), and be used as a building block for protein synthesis. Considering the competitive pathways existing at L-serine node, we firstly overexpressed the L-serine deaminase to enhance the conversion of
L-serine into pyruvate. In E. coli, L-serine is deaminated by three L-serine deaminases, which are encoded by $s d a A$, $s d a B$ and $t d c G$, respectively. L-serine deaminases I (SdaA) which is encoded by $s d a A$ gene, is responsible for L-serine degradation in minimal media [14]. Moreover, a previous report has shown that sdaA-overexpressing Corynebacterium glutamicum could grow in the medium using Lserine as the sole carbon source [17]. Thus, in order to activate the SD pathway, $s d a A$ was selected to be overexpressed by replacing the native $s d a A$ promoter with a strong constitutive promoter trc in JM109, resulting in strain SD01 (JM109, $\mathrm{P}_{t r c}-s d a A$ ). To test the effect of overexpressing sdaA on PHB production, SD01 and JM109 were both transformed with the plasmid pBHR68 which consists of the PHB biosynthesis genes from Ralstonia eutropha, creating strains SD01 (pBHR68) and JM109 (pBHR68). The PHB production of SD01 (pBHR68) was $3.58 \mathrm{~g} / \mathrm{L}, 1.72$-fold of that of JM109 (pBHR68) (Figure 2), and the PHB content is $73.8 \%$ of the cell dry weight (CDW) (Figure 2). These results suggested that overexpression of sdaA obviously pulled more L-serine to pyruvate and led to the improvement of PHB production. Thus, SD01 was chosen as the host for subsequent modifications to improve PHB production.

\section{Influence of gene overexpression in L-serine biosynthetic pathway on PHB production}

It is reasonable that efficient conversion of L-serine to pyruvate requires sufficient availability of L-serine. To enhance the biosynthesis of L-serine, we overexpressed the genes of de novo L-serine biosynthetic pathway. Lserine is synthesized from D-3-phosphoglycerate by three reactions catalyzed by D-3-phosphoglycerate dehydrogenase, D-3-phosphoserine aminotransferase and phosphoserine phosphatase, which are encoded by $\operatorname{ser} A, \operatorname{ser} C$ and ser $B$, respectively (Figure 1). D-3-phosphoglycerate dehydrogenase is regulated by allosteric end-product inhibition. Moreover, a published report has showed that a truncated D-3-phosphoglycerate dehydrogenase (PGDH) $\operatorname{ser} A^{\Delta 197}$ was no longer inhibited by L-serine in C. glutamicum [18]. As such, we combined ser $A^{\Delta 197}$ together with $\operatorname{ser} B$ and $\operatorname{ser} C$ into an artificial operon driven by the constitutive promoter trc, creating plasmid pTSer. Strain SD01 was transformed with plasmid pTSer for activating the Serine-Deamination (SD) pathway. After 48h cultivation, $3.96 \mathrm{~g} / \mathrm{L}$ PHB was accumulated by SD01 (pBHR68/ pTSer), which was only slightly higher than that of the reference strain SD01 (pBHR68/pTrc101) (Figure 2).

However, the strategy of overexpressing ser $A B C$ mainly led to improvement of cell growth, rather than specific PHB accumulation (Figure 2). This phenomenon might be due to the difference between the kinetic parameters of the two enzymes, SHMT and SdaA. The $\mathrm{K}_{\mathrm{m}}$ value for Lserine of SdaA is higher than that of SHMT (2.67 vs 0.3 


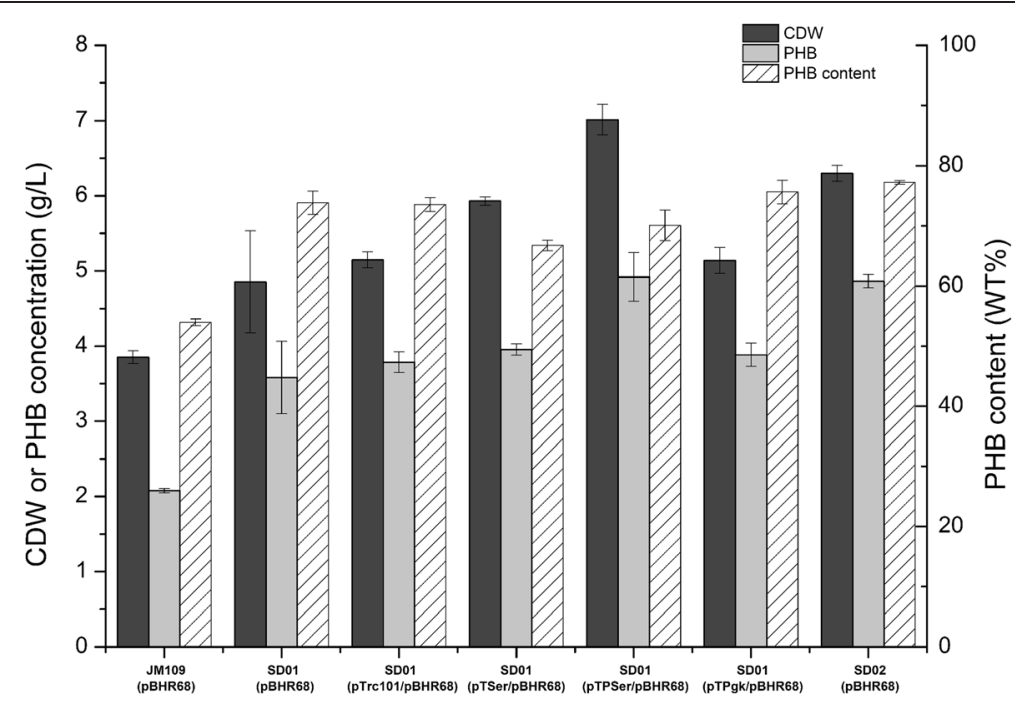

Figure 2 Comparison of CDW, PHB concentration and PHB content in recombinant $E$. coli strains. Histogram shows the mean of three biological replicates, and error bars show standard deviations.

$\mathrm{mM}[19,20])$. The overexpression of $\operatorname{ser} A B C$ improved the availability of L-serine, which contributed much more to the reaction catalyzed by SHMT than the reaction catalyzed by SdaA. SHMT is the main source of $\mathrm{C} 1$ carbon and glycine for cell growth in E.coli, so residual cell dry weight was increased under this condition [21,22].

On the other hand, the $\mathrm{k}_{\mathrm{cat}}$ value of SdaA is much higher than that of SHMT (436 vs $\left.5 \mathrm{~s}^{-1}[19,20]\right)$, and the corresponding $\mathrm{k}_{\mathrm{cat}} / \mathrm{K}_{\mathrm{m}}$ values are 163.3 and $16.7 \mathrm{mM}^{-1} \mathrm{~s}^{-1}$, respectively. This means only when the intracellular concentration of L-serine is further improved to some extent, the serine deamination reaction can obviously overwhelm the competing reaction catalyzed by SHMT. Previous researches have shown that $p g k$ (encodes phosphoglycerate kinase) overexpression could divert carbon flux into the D-3-phosphoglycerate pool and further promote the biosynthesis of L-serine [23]. To push more flux into the SD pathway, $p g k$ gene was ligated into pTSer, creating plasmid pTPSer. The PHB production of SD01 (pBHR68/pTPSer) reached $4.92 \mathrm{~g} / \mathrm{L}$, increasing by $30.2 \%$ compared to that of SD01 (pBHR68/pTrc101) harboring the empty vector (Figure 2). However, when $p g k$ was overexpressed alone, the $\mathrm{PHB}$ production of SD01 (pBHR68/pTPgk) showed no significant change, compared with that of SD01 (pBHR68/pTrc101). These results suggested that activating the SD pathway by cooverexpressing the L-serine deaminase, enzymes in Lserine biosynthesis pathway and phosphoglycerate kinase had a significant effect on PHB production.

Considering the instability of plasmid system and the metabolic burden resulted from plasmid replication $[24,25]$, the $p g k-\operatorname{ser} A B C$ operon together with the fragment Trc-162 was integrated into the choromosome of SD01 at $\operatorname{serC}$ site, creating strain SD02 (SD01, $\mathrm{P}_{\text {Trc-162- }}$
Ser $A B C$ ). The transcription levels of genes in SD pathway in strain SD02 were compared with that of the reference strain JM109 through RT-PCR analysis. As shown in Figure 3A, the genes of SD pathway have been successfully overexpressed. SD02 (pBHR68) accumulated $4.86 \mathrm{~g} / \mathrm{L} \mathrm{PHB}$, which was almost the same as that of SD01 (pBHR68/pTPSer), but had a higher PHB content of $77.2 \%$ CDW (Figure 2). Therefore SD02 was selected for further engineering.

\section{Effect of combining gene overexpression in ED pathway} with the SD pathway on PHB production

Redirecting the carbon metabolism into the ED pathway in the engineered strain is beneficial to PHB production, since it avoids the carbon loss and balance the NADPH need $[12,26]$. Moreover, enhancing ED pathway might increase the availability of pyruvate and further enrich the in vivo pool of acetyl-CoA. As such, we tried to test the effect of enhancing the ED pathway on PHB accumulation.

The ED pathway joins the oxidative pentose phosphate pathway to EMP pathway via two enzyme-catalyzed reactions. The two critical enzymes are phosphogluconate dehydratase and 2-keto-3-deoxygluconate 6-phosphate aldolase, which were encoded by edd and eda, respectively. In $E$. coli, the ED pathway has been shown to be inactive with glucose as the carbon source [27]. Thus, the upstream regulated region of the edd-eda operon in SD02 was replaced with a constitutive promoter J23119 (http:// partsregistry.org/Part:BBa_J23119) for eliminating the repression of the ED pathway and activating the ED pathway, resulting in SD03 (SD02 $\mathrm{P}_{J 23119}$-edd). Unexpectedly, strain SD03 (pBHR68) produced $2.90 \mathrm{~g} / \mathrm{L}$ PHB which was a significant decrease compared with that of SD02 (pBHR68) (Table 1). Then fragment Trc-162 was inserted 


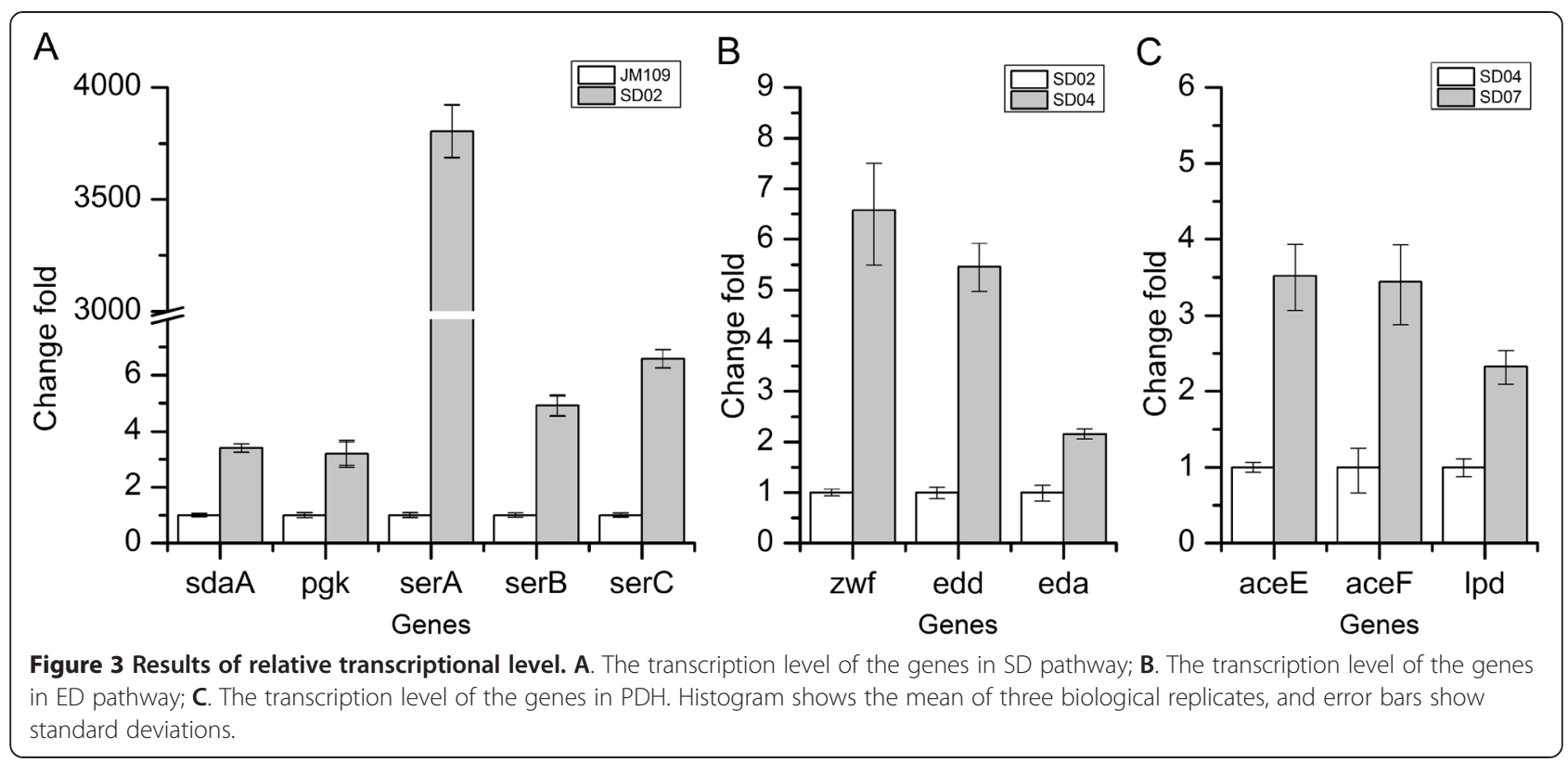

at the upstream of $z w f$ gene in SD03 to further enhance the flux of ED pathway, creating strain SD04 (SD03, $\mathrm{P}_{\text {Trc- }}$

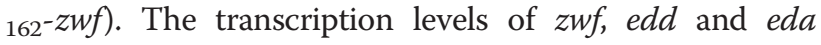
genes in SD04 were up-regulated by 5.46, 4.57 and 2.16fold, respectively (Figure $3 \mathrm{~B}$ ). PHB was accumulated to $3.39 \mathrm{~g} / \mathrm{L}$ in SD04 (pBHR68), a 16.9\% increase compared with that of SD03 (pBHR68), but was still lower than that of the parent strain SD02 (pBHR68) (Table 1). In addition, a small amount of pyruvate and acetate had been detected in the media of strains SD03 (pBHR68) and SD04 (pBHR68), but not detected in SD02 (pBHR68) (Table 1). This might be due to the improvement of glucose consumption after co-overexpressing the edd, eda, zwf genes (Table 1), which resulted in pyruvate accumulation in the medium. Moreover, the acetate was produced directly from pyruvate by pyruvate oxidase (encoded by poxB) in the JM109 derivative strains [28]. Thus it was possible that the accumulation of pyruvate and acetate retarded the cell growth and led to lower PHB production, although the intracellular acetyl-CoA concentration and the [NADPH]/ $\left[\mathrm{NADP}^{+}\right]$ratio was increased (Figure 4 , Table 2 ). So we tried to enhance the conversion of pyruvate to acetyl-CoA to eliminate the pyruvate accumulation and increase the $\mathrm{PHB}$ production.

\section{Overexpression of the pyruvate dehydrogenase complex} for enhanced PHB production

In $E$. coli, pyruvate is metabolized by the pyruvate dehydrogenase $(\mathrm{PDH})$ complex and pyruvate oxidase during aerobic growth conditions [15,29]. To enhance the expression of PDH complex, fragment Trc-162 was inserted into the upstream of the PDH operon in SD02 and SD04 as mentioned in the methods section, resulting in strains SD06 (SD02, $\mathrm{P}_{\text {Trc-162-aceEF) and SD07 }}$

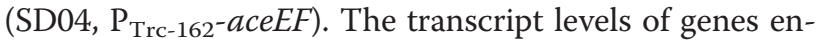
coding the PDH complex were presented in Figure $3 \mathrm{C}$,

Table 1 Cell growth, PHB production, and by-products formation by strains study at the end of the cultivation

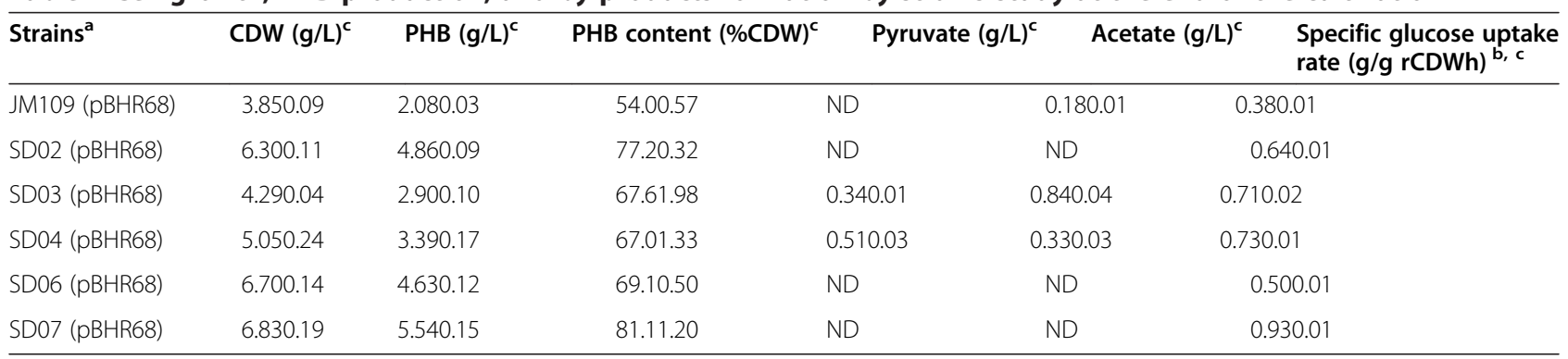

${ }^{\mathrm{a}}$ strains were cultured for $48 \mathrm{~h}$ in mineral salt medium supplemented with $1 \mathrm{~g} / \mathrm{L}$ yeast exact and $20 \mathrm{~g} / \mathrm{L}$ glucose at $37 \mathrm{C}$ at a rotation rate of $220 \mathrm{rpm}$ under aerobic conditions;

${ }^{\mathrm{b}}$ The specific glucose uptake rate was determined between $0 \mathrm{~h}$ and $24 \mathrm{~h}$ of the fermentation. $\mathrm{rCDW}$ : residual cell dry weight.

${ }^{\mathrm{C}}$ Data were expressed as average values and standard deviations (SD) of three parallel studies. 


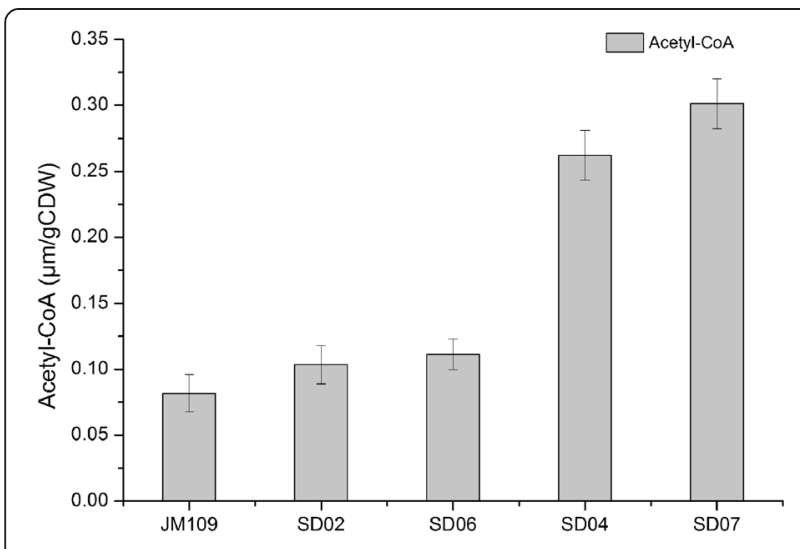

Figure 4 Intracellular acetyl-CoA concentrations of recombinant E. coli. The average cell dry weight for all of the strains was $0.38 \mathrm{~g} /$ liter per optical-density $\left(\mathrm{OD}_{600}\right)$ unit of culture. Histogram shows the mean of three biological replicates, and error bars show standard deviations.

which indicated that the genes of the PDH complex were successfully overexpressed. The PHB production of SD06 (pBHR68) was $4.63 \mathrm{~g} / \mathrm{L}$ (69.1\% CDW), no significant change compared with the reference strain SD02 (pBHR68) (Table 1), as well as the intracellular concentration of acetyl-CoA (Figure 4).

In contrast, the PHB concentration of the strain SD07 (pBHR68) was $5.54 \mathrm{~g} / \mathrm{L}$ and PHB content was $81.1 \%$ $\mathrm{CDW}$, significantly increased from $2.90 \mathrm{~g} / \mathrm{L}$ of the parent strain SD04 (pBHR68). As expected, no trace of pyruvate and acetate was detected in the medium of SD07 (pBHR68) (Table 1), and the intracellular acetyl-CoA concentration of SD07 was further increased compared with that of SD04 (Figure 4). These indicated that the diversion of excess pyruvate to acetyl-CoA enhanced the availability of acetyl-CoA for PHB synthesis, and thus rebalanced the flux distribution at the pyruvate node.

PHB fermentation using other unrelated carbon sources Xylose is a major constituent of lignocellulose biomass, and glycerol is a major byproduct of petrochemical

Table 2 Comparison of the intracellular NADP ${ }^{+}$, NADPH concentrations and [NADPH]/[NADP+] ratios of the recombinant strains without the $\mathrm{PHB}$ synthesis genes

\begin{tabular}{|c|c|c|c|}
\hline Strains $^{a}$ & $\begin{array}{l}\text { NADP }^{+} \\
(\mu \mathrm{mol} / \mathrm{gCDW})^{\mathrm{b}, \mathrm{c}}\end{array}$ & $\begin{array}{l}\text { NADPH } \\
(\mu \mathrm{mol} / \mathrm{gCDW})^{\mathrm{c}}\end{array}$ & $\begin{array}{l}{[\mathrm{NADPH}] /\left[\mathrm{NADP}^{+}\right]} \\
\text {ratio }^{c}\end{array}$ \\
\hline JM109 & 0.990 .02 & 0.720 .02 & 0.730 .02 \\
\hline SD02 & 1.000 .02 & 0.730 .01 & 0.730 .01 \\
\hline SD04 & 0.910 .04 & 0.840 .03 & 0.920 .01 \\
\hline SD07 & 0.860 .02 & 0.770 .03 & 0.900 .01 \\
\hline
\end{tabular}

${ }^{\mathrm{a} C e l l s}$ were cultivated in MS medium containing $10 \mathrm{~g} / \mathrm{L}$ of glucose at $37 \mathrm{C}$ for $6 \mathrm{~h}$.

${ }^{\mathrm{b}} \mathrm{CDW}$ The average cell dry weight for all of the strains was $0.38 \mathrm{~g} /$ liter per optical-density $\left(\mathrm{OD}_{600}\right)$ unit of culture.

'The data shown are the average and standard deviations of three parallel experiments. products, both of which have been utilized for the production of various PHAs as cheap carbon sources [30-32]. We also tested the PHB production performance of the recombinant strains by using xylose or glycerol as carbon sources. Compared to wild type strain, SD06 (pBHR68) and SD07 (pBHR68) showed significant increase in PHB production and PHB content (Table 1). However, no significant difference was observed between these two strains when xylose or glycerol was served as the sole carbon source (Table 3). The reason for this phenomenon was that xylose or glycerol turned into D3-phosphoglyceraldehyde via a series of metabolic reactions and flow into the EMP pathway or SD pathway rather than the ED pathway under aerobic conditions. These results suggested that the strategy reported here should be useful for the bio-based production of PHB from different carbon sources.

\section{Conclusions}

Through combined engineering of SD pathway, ED pathway and $\mathrm{PDH}$, a recombinant E. coli strain was obtained which led to significantly enhanced PHB accumulation. The final strain produced $5.52 \mathrm{~g} / \mathrm{L}$ PHB from glucose with a content of $81.11 \%$ CDW. A similar phenomenon was observed when xylose or glycerol was served as carbon sources. In conclusion, enhancing the availability of acetylCoA via engineering the SD pathway, ED pathway and the $\mathrm{PDH}$ complex offered an effective way for improving the $\mathrm{PHB}$ production in $E$. coli from different carbon sources.

\section{Methods}

Bacterial strains, primers, and plasmids construction

Bacterial strains and plasmids used in this study were listed in Table 4. E. coli DH5 $\alpha$ was used for plasmid construction. The truncated $\operatorname{ser} A^{\Delta 197}$ from C. glutamicum and $\operatorname{ser} B$, serC genes from $E$. coli with the synthesized ribosome binding sites (RBSs) were amplified by polymerase chain reaction (PCR) using the primers serAF/serAR,

Table 3 PHB accumulation of the strains in xylose or glycerol

\begin{tabular}{lllll}
\hline Carbon source & Strains $^{\mathbf{a}}$ & $\begin{array}{l}\text { CDW } \\
(\mathbf{g} / \mathbf{L})^{\mathbf{b}}\end{array}$ & $\begin{array}{l}\text { PHB } \\
(\mathbf{g} / \mathbf{L})^{\mathbf{b}}\end{array}$ & $\begin{array}{l}\text { PHB content } \\
{\text { (\%CDW })^{\mathbf{b}}}^{(2.05}\end{array}$ \\
\hline Xylose & JM109(pBHR68) & 3.760 .09 & 1.790 .05 & 47.50 .86 \\
Xylose & SD06(pBHR68) & 5.720 .05 & 3.900 .06 & 68.11 .00 \\
Xylose & SD07(pBHR68) & 5.700 .13 & 3.920 .04 & 68.81 .06 \\
Glycerol & JM109(pBHR68) & 3.380 .08 & 1.370 .06 & 38.41 .23 \\
Glycerol & SD06(pBHR68) & 3.910 .05 & 2.150 .10 & 54.91 .72 \\
Glycerol & SD07(pBHR68) & 3.790 .07 & 2.120 .08 & 56.01 .03 \\
\hline
\end{tabular}

${ }^{a}$ Bacteria were cultured for $48 \mathrm{~h}$ in mineral salt medium supplemented with 1 $\mathrm{g} / \mathrm{L}$ yeast exact and $20 \mathrm{~g} / \mathrm{L}$ xylose/glycerol at $37 \mathrm{C}$ at a rotation rate of 220 rpm under aerobic conditions.

${ }^{b}$ Data were expressed as average values and standard deviations (SD) of three parallel studies. 
Table 4 Strains and plasmids used in this study

\begin{tabular}{|c|c|c|}
\hline Strain or plasmid & Relevant genotype $^{\text {b }}$ & Source or reference \\
\hline \multicolumn{3}{|l|}{ Strains } \\
\hline E. coli DH5a & $\begin{array}{l}\text { Coli Genetic Stock Center } \\
\text { strain (CGSC) No. } 12384\end{array}$ & $\operatorname{CGSC}^{a}$ \\
\hline E. coli JM109 & $\begin{array}{l}\text { recA1, endA1, gyrA96, thi, } \\
\text { hsdR17, supE44, relA1, } \\
\Delta(\text { lac proAB)/F [traD36, } \\
\left.\text { proAB } B^{+}, l^{9} c^{9} \text { lac } Z \Delta \mathrm{M} 15\right]\end{array}$ & TaKaRa (Dalian, China) \\
\hline SD01 & SD01, $P_{\text {sdaA }}:$ Ptrc & This study \\
\hline SD02 & $\begin{array}{l}\text { SD02, SerC:: Ptrc-pgk- } \\
\text { SerABC }\end{array}$ & This study \\
\hline SD03 & $\mathrm{SD02}, \mathrm{P}_{\text {edd }}: \mathrm{P}_{J 23119}$ & This study \\
\hline SD04 & $\begin{array}{l}\text { SD02, } P_{\text {edd-eda }}: \mathrm{P}_{J 23119}, \mathrm{P}_{\text {zwff: }} \\
\mathrm{P}_{\mathrm{Trc}-162}\end{array}$ & \\
\hline SD06 & SD02, $P_{\text {aceE }}: \mathrm{P}_{\text {Trc-162 }}$ & This study \\
\hline SD07 & SD04, $P_{\text {aceE }}: \mathrm{P}_{\text {Trc-162 }}$ & This study \\
\hline Tet-Trc-162 & JM109, tetA-trc-M1-162-glyA & Lab collection \\
\hline \multicolumn{3}{|l|}{ Plasmids } \\
\hline pTrc101 & $\begin{array}{l}\text { Expression vector, pSC101 } \\
\text { replication, constitutive } \\
\text { trc promoter, } \mathrm{Cm}^{r}\end{array}$ & This study \\
\hline pTSer & $\begin{array}{l}\text { pSC101 replication, } \mathrm{Cm}^{\mathrm{r}} \text {, } \\
\text { Ptrc -serA-serB-serC }\end{array}$ & This study \\
\hline pTPSer & $\begin{array}{l}\text { pSC101 replication, } \mathrm{Cm}^{\mathrm{r}} \text {, } \\
\mathrm{P}_{\text {trc }}{ }^{-} \text {pgk-serA-serB-serC }\end{array}$ & This study \\
\hline pTPgk & $\begin{array}{l}\text { pSC101 replication, } \mathrm{Cm}^{\mathrm{r}} \text {, } \\
\mathrm{P}_{\text {trc-pgk }}\end{array}$ & This study \\
\hline pBHR68 & $\begin{array}{l}\text { pBluscript SK(-) derivative, } \\
p h b A_{R e}, p h b B_{R e}, p h b C_{R e} \\
\text { cloned from } R \text {. eutropha }\end{array}$ & [34] \\
\hline pTKRED & $\begin{array}{l}\text { pSC101 replication, } \\
\text { temperature sensitive } \\
\text { replication origin, SpCr, } \\
\text { ParaBAD-driven I-Scel gene, } \\
\text { Red recombinase expression } \\
\text { plasmid, lac-inducible } \\
\text { expression }\end{array}$ & {$[35]$} \\
\hline $\mathrm{pTKS} / \mathrm{CS}$ & $\begin{array}{l}\text { p15A replication, } \mathrm{Cm}^{r} \text {, Tet', } \\
\text { I-Scel restriction sites }\end{array}$ & [35] \\
\hline
\end{tabular}

${ }^{\mathrm{a}}$ Coli Genetic Stock Center.

${ }^{\mathrm{b}}$ Abbreviations: Amp ampicillin, Cm chloramphenicol, Tet tetracycline, Spc spectinomycin, $r$ resistance.

serBF/serBR and serCF/serCR, respectively. Primers pTrc101R/pTrc101F were used to amplify the backbone of the plasmid pTrc101. The ser $A B C$ genes were cloned into pTrc101 by circular polymerase extension cloning (CPEC) [33], resulting in plasmid pTSer. The $p g k$ gene was amplified with the primers $\mathrm{pgkF} / \mathrm{pgkR}$ from $E$. coli genome and ligated into the pTSer and pTrc101, creating pTPSer and pTpgk. E.coli strains were transformed with the plasmids for PHB production or activate the SD pathway, for example, JM109 (pBHR68) represent the JM109 haboring the pBHR68 plasmid.

\section{Genome replacement manipulation}

The DNA fragment insertion or replacement strains were constructed by using the method reported by Lin et al with appropriate modifications [36]. The strategies of fragment construction were outlined in Figure 5. The final fragments were transformed into the competent cells with expression of the $\lambda$ red recombination enzymes. The tetracycline resistant mutants were screened and confirmed by colony PCR. To induce I-SceI endonuclease expression and remove the resistance gene tet $\mathrm{A}$ from the genome, the positive colony was inoculated into $5 \mathrm{ml}$ of LB medium with $100 \mu \mathrm{g} / \mathrm{mL}$ spectinomycin, $2 \mathrm{mM}$ isopropyl- $\beta$-D-thiogalactopyranoside (IPTG), and $0.2 \% \mathrm{w} / \mathrm{v} \mathrm{L}$ arabinose. After overnight cultivation, cultures were diluted to appropriate concentration and plated on LB agar plates. The loss of tetA was confirmed by colony PCR. The technological process in detail was displayed in Figure 5. Primers used were listed in Table 5.

For swapping the promoter of $z w f, \mathrm{PDH}$ complex operon and $p g k-s e r A B$ fragment insertion, the tet $A$ fragment was amplified from strain Tet-Trc-162, and fused with the up and low homologous flanks (Figure 5C). The low flank of $p g k$-serABC fragment was amplified from the plasmid pTPSer (Figure 5D). Fragment Trc-162 consisted of the trc promoter core sequence and M1-162 [37] in tandem.

\section{Cultivation conditions}

During strains and plasmids construction, cultures were grown at $30 \mathrm{C}$ or $37 \mathrm{C}$, in Luria broth (per liter: $10 \mathrm{~g}$ tryptone, $5 \mathrm{~g}$ yeast extract, and $10 \mathrm{~g} \mathrm{NaCl}$ ) with or without agar (2\%) as indicated. Minimal sodium medium (MS medium) with $1 \mathrm{~g} / \mathrm{L}$ yeast extract was used as seed culture and shake flask medium which contained (in grams per liter): glucose/xylose/glycerol 20.0, $\left(\mathrm{NH}_{4}\right)_{2} \mathrm{SO}_{4}$ 2.0, $\mathrm{MgSO}_{4} 7 \mathrm{H}_{2} \mathrm{O}$ 0.4, $\mathrm{Na}_{2} \mathrm{HPO}_{4}$ 3.83, $\mathrm{KH}_{2} \mathrm{PO}_{4}$ 1.5, Fe (III)- $\mathrm{NH}_{4}$-citrate $0.05, \mathrm{CaCl}_{2} 0.02$, and $1 \mathrm{~mL} / \mathrm{L}$ trace element [38]. When necessary, a final concentration of $10 \mu \mathrm{g} / \mathrm{mL}$ chloromycetin and/or $100 \mu \mathrm{g} / \mathrm{mL}$ ampicillin were added. Colonies were inoculated into $5-\mathrm{ml} \mathrm{LB}$ culture medium and grown at $37 \mathrm{C}$ with shaking overnight. Then the culture was inoculated with $1 \%$ into $250-\mathrm{ml}$ flask with $50 \mathrm{ml}$ culture medium as seed culture grown at $37 \mathrm{C}$ in MS medium for $12 \mathrm{~h}$ at $220 \mathrm{rpm}$ on a rotary shaker. Seed culture was then inoculated into $500-\mathrm{mL}$ flask with $100 \mathrm{ml}$ culture medium (with the initial $\mathrm{OD}_{600}$ of 0.04 ) and grown at $37 \mathrm{C}$ and $220 \mathrm{rpm}$ on a rotary shaker for $48 \mathrm{~h}$. Three biological replicates were performed to detect the accumulation of PHB. To assess the significant difference of the PHB accumulation, data was subject to Students $\mathrm{t}$ test analysis with $p<0.05$ being significantly different. 
Table 5 Primer sequence used in this study

\begin{tabular}{|c|c|}
\hline Primer name & Primer sequence $(5 \rightarrow 3)$ \\
\hline serAF & $\begin{array}{l}\text { TCTAGAGTCGAGCACAGCCGTATTCTAAGGAGGTCAAATGTGAGCCAGAATGGCCGTCC } \\
\end{array}$ \\
\hline serAR & GTAGTITGTCTCCGTTIAATTAGTCTTTAAGATCTITAGCGAGCCAGATCCATCCACA \\
\hline serBF & CGCTAAAGATCTTAAAGACTAATTAAACGGAGACAAAACTACATGCCTAACATTACCTG \\
\hline serBR & CCTCCTTTATAAAATAGATTACTACCTTGGATCCTTACTTCTGATTCAGGCTGCCTGAG \\
\hline serCF & GTAAGGATCCAAGGTAGTAATCTATTTATAAAGGAGGAGGTAATGGCTCAAATCTTCA \\
\hline $\operatorname{serCR}$ & CTTGCATGCCTGCAGGTCGACTTAACCGTGACGGCGTTCG \\
\hline pTrc101R & TCTAGAGGATCCCCGGGTACCGAGCTC \\
\hline pTrc101F & TCATGGTTGAGTTCGAACGCCGTCACGGTTAAGTCGACCTGCAGGCATGCAA \\
\hline pgk_F & TCGAGCTCCGCACCACAAAAACGACACCATAGGGGGGCAAACGATGTCTGTAATTAAGA \\
\hline pgk_R & GTGCGGAGCTCTTACTTCTTAGCGCGCTCTT \\
\hline \multicolumn{2}{|c|}{ Primers for genetic manipulation } \\
\hline SdaAp U_F & TATACCGCCTTCCGCCGTTG \\
\hline SdaAp U_R & AATGAGCCGGATGATTAATTGTCAACAGCTCATTAGTCAGGGTTTCACACCAA \\
\hline SdaAp T_F & ATGAGCTGTTGACAATTAATCATCCGGCTCATTACCCTGTTATCCCTACTAAGC \\
\hline SdaAp T_R & $\begin{array}{l}\text { TACTCCGTCGACTGTGTGACCACACATTATACGAGCCGGATGATTAATTGTCAACAGCTCATTAGGGATAACAG } \\
\text { GGTAATGTACC }\end{array}$ \\
\hline SdaAp L_F & ATAATGTGTGGTCACACAGTCGACGGAGTAACGACTCTCGTAAATAAGGAGTTTAAAGTGATTAGTCTATTCGACAT \\
\hline SdaAp L_R & AAACCGGGAATACTGTCGAT \\
\hline Tet $\mathrm{U}$ & AGCTGAGTCAGGAGATGCGG \\
\hline Tet $\mathrm{R}$ & AGCTGTTTCCTGGTTTAAAC \\
\hline Ser U_F & CTGGTCGAAACTCAATAACTCC \\
\hline Ser U_R & TTAATTGTCAACAGCTCATCCGCATCTCCTGACTCAGCTTTCCCCTCACCACGTTGCGT \\
\hline Ser L_F & GTACAGTACTTCAATTTGTTAAAACCAGGAAACAGCTATGTCTGTAATTAAGATGACCG \\
\hline Ser L_R & AGCATTATCAGAGAGTTGCCAT \\
\hline PaceEF U_F & GCCAGAACTTCGAATTGCTC \\
\hline PaceEF U_R & TGTCAACAGCTCATCCGCATCTCCTGACTCAGCTGGGTTATTCCTTATCTATC \\
\hline PaceEF L_F & GTACAGTACTTCAATTTGTTTAAACCAGGAAACAGCTATGTCAGAACGTTTCCCAA \\
\hline PaceEF L_R & CCAGTTCCAGATTACCCGGAT \\
\hline Pzwf U_F & TCCGCACTGAAAGAAATCGAA \\
\hline Pzwf U_R & TGTCAACAGCTCATCCGCATCTCCTGACTCAGCTCGCATTCTCCTTAAGTTAAC \\
\hline Pzwf L_F & GTACAGTACTTCAATTTGTTAAACCAGGAAACAGCTATGGCGGTAACGCAAACAG \\
\hline Pzwf L_R & ATTCAGTITTGCCTCGCCAAG \\
\hline Pedd U_F & CAGAAACCTITAATCAGACGCATC \\
\hline Pedd U_R & ACAGGGTAATCTAGGACTGAGCTAGCTGTCAACCATAAAGGATAAGCGCAGAT \\
\hline Pedd T_F & CTITATGGTTGACAGCTAGCTCAGTCCTAGATTACCCTGTTATCCCTACTAAGCACT \\
\hline Pedd T_R & TATACCTAGGACTGAGCTAGCTGTCAACCATAAAGAAGGGATAACAGGGTAATGTACCA \\
\hline Pedd L_F & ACAGCTAGCTCAGTCCTAGGTATAATGCTAGCACGAACAGGCGTTTCAGTCATAAATCC \\
\hline Pedd L_R & CGCAACATGCTITTCAAAGAGG \\
\hline \multicolumn{2}{|c|}{ Primers for RT-PCR } \\
\hline RTsdaA_F/R & CTATGAAGGCAGGTAAACAG/CGAGTAACGCTATCCAGTA \\
\hline RTpgk_F/R & GTTCTAAAGTATCTACCAAACTG/ATACCACCACCAACAATC \\
\hline RTSerA_F/R & CGATGGTGAG TGGAAACGCT/CGTAGGCAAGGATCTCATCA \\
\hline RTSerB_F/R & GAGATCATGGACGGTAAA/ CAGAGTTTTCGCTTTGTA \\
\hline RTSerC_F/R & TATTCCATCCTCAACGATA/GACCAGACCAGATAGATA \\
\hline
\end{tabular}


Table 5 Primer sequence used in this study (Continued)

\begin{tabular}{ll}
\hline RTzwf_F/R & TTGCTAACTCCCTGTTTGT/CTTCTTCTGCCACGGTAA \\
RTaceE_F/R & TTACGAAGTTGCTGTCAT/AGCGTAGTGATGTAGTAGTA \\
RTaceF_F/R & AAATCCTGGTGAAAGTTG/TCCATAGAAGCCTTGTC \\
RTIpd_F/R & CAGCAAGAAATTCAACCT/CCTTCCATCGTCACATAA \\
RTrrsA_F/R & TACGACCAGGGCTACACACG/ATCCGGACTACGACGCACTT
\end{tabular}

\section{Analytical techniques}

The growth of cell was monitored by measuring the $\mathrm{OD}_{600}$ with an ultraviolet spectrophotometer (Beijing Puxi Universal Co Ltd). Glucose in the fermentation broth was determined utilizing a SBA sensor machine (Institute of Microbiology, Shangdong, China). Bacteria were harvested by centrifugation at 8,000 $\mathrm{g}$ for $10 \mathrm{~min}$ and then washed with distilled water. Cell dry weight (CDW) was measured after lyophilization and vacuum drying. PHB content was analyzed by gas chromatography (Persee, China) with an Agilent J\&W Capillary GC column after methanolysis of lyophilized cells in chloroform. To determine the concentration of pyruvate, acetate, glycerol and xylose, culture samples were centrifuged at 12,000 $\mathrm{g}$ for $5 \mathrm{~min}$ and the aqueous supernatant used for HPLC analysis on an Agilent 1100 Series HPLC system equipped with an Aminex HPX-87H anion exchange column (Bio-Rad Laboratories, Richmond, CA, USA) and refractive index detector. A mobile phase of $5 \mathrm{mM} \mathrm{H}_{2} \mathrm{SO}_{4}$ at a $0.4 \mathrm{~mL} / \mathrm{min}$ flow rate was used.

For the determination of intracellular acetyl-CoA, 40 $\mathrm{mL}$ mid-exponential phase cell culture was taken into precooled centrifuge tubes and centrifuged at $8000 \mathrm{~g}$ and $4 \mathrm{C}$ for $10 \mathrm{~min}$. The cell pellets were washed with $40 \mathrm{~mL}$ $100 \mathrm{mM}$ Tris- $\mathrm{HCl}$ buffer ( $\mathrm{pH}$ 8.0). Acetyl-CoA was analyzed by HPLC as previous reported $[39,40]$. For determination of intracellular $\mathrm{NADP}^{+}$and NADPH, $10 \mathrm{~mL}$ mid-exponential phase cell culture was taken into precooled centrifuge tubes and centrifuged at $8000 \mathrm{~g}$ and $4 \mathrm{C}$ for $10 \mathrm{~min}$. The intracellular $\mathrm{NADP}^{+}$and $\mathrm{NADPH}$ were analyzed by HPLC as previous reported [41].

\section{Quantitative real-time reverse transcription (RT)-PCR analysis}

The recombinant strains harboring pBHR68 plasmid were cultured with the same fermentation media and culture condition with $1 \%(\mathrm{w} / \mathrm{v})$ glucose. Cells were harvested when $\mathrm{OD}_{600}$ reached 1 . Total mRNA were extracted using the RNAprep pure Cell/Bacteria Kit (Tiangen, Beijing, China) as described by the manufacturer. The cDNA was amplified using FastQuant RT Kit (Tiangen, Beijing, China) with the total mRNA as the templates. Samples were then analyzed using a Light

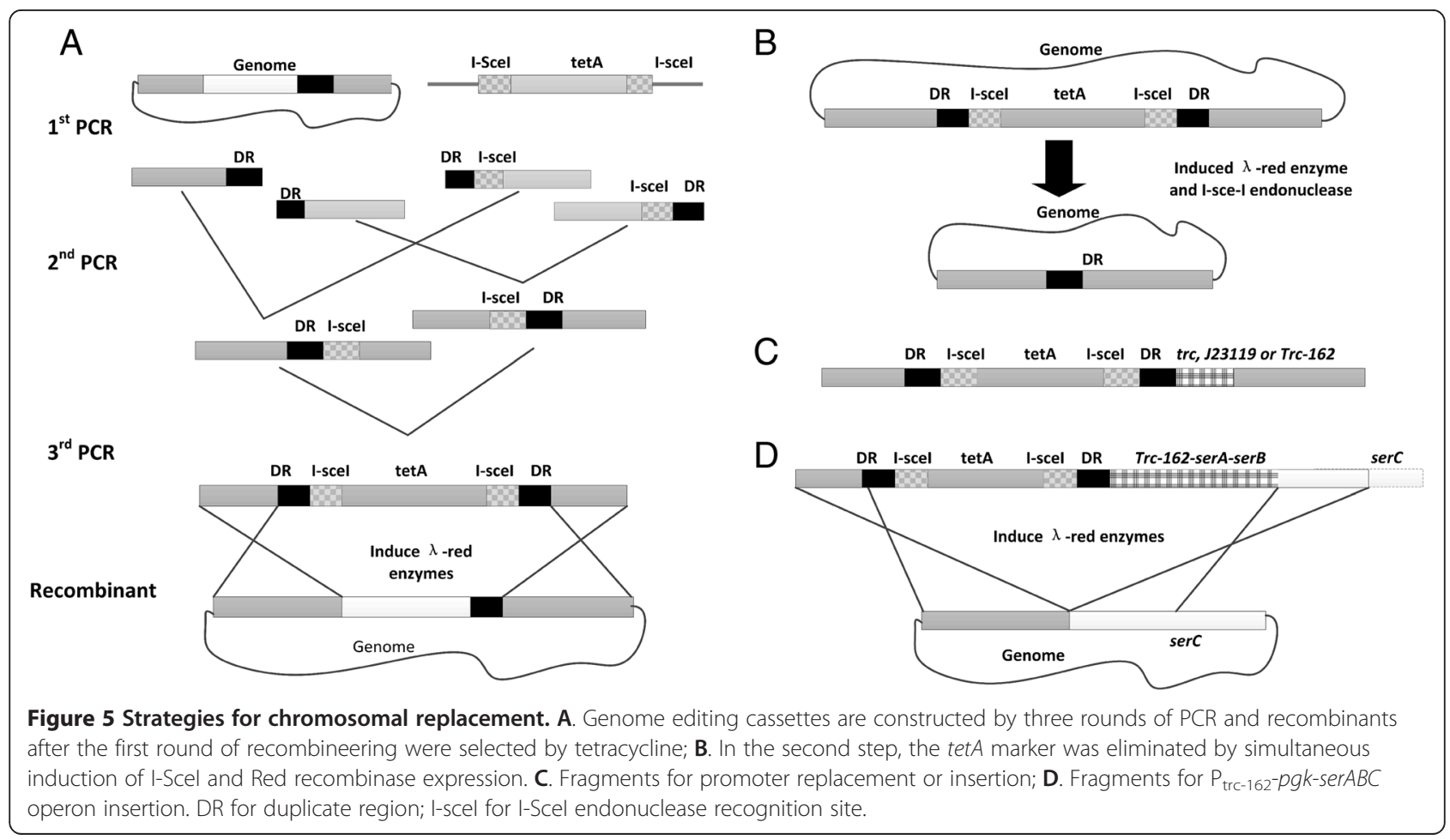


Cycler 480 II (Roche, Basel, Switzerland) with RealMasterMix (SYBR Green I) (Tiangen, Beijing, China). Quantity real-time PCR (qPCR) amplification primers were designed and were listed in Table 5. The rrsA gene was selected as internal standard for normalization and three biological replicates were performed. The obtained data were analyzed by using the $2^{-\Delta \Delta \mathrm{Ct}}$ method described previously [42].

\section{Competing interests}

The authors declare that they have no competing interests.

\section{Authors contributions}

YZ, ZQL and TC designed the experiments; YZ, ZQL, QJL, YFL and ZWW performed the experiments; YZ, ZQL, TC and HWM wrote the manuscript; TC and XMZ supervised the work; and all authors contributed to the discussion of the research. All authors read and approved the final manuscript.

\section{Acknowledgments}

We thank Prof. Guo-Qiang Chen (Tsinghua University) for kindly providing the plasmid pBHR68. We thank Dr. Thomas E. Kuhlman for kindly providing requested plasmids. This work was supported by National 973 Project [2012CB725203, 2011CBA00804]; National Natural Science Foundation of China [NSFC-21176182, NSFC-21206112, NSFC-21390201]; and National High-tech R\&D Program of China [2012AA02A702, 2012AA022103].

\section{Author details}

${ }^{1}$ Key Laboratory of Systems Bioengineering (Ministry of Education), Tianjin University, Tianjin 300072, Peoples Republic of China. ${ }^{2}$ SynBio Research Platform, Collaborative Innovation Center of Chemical Science and Engineering (Tianjin), School of Chemical Engineering and Technology, Tianjin University, Tianjin 300072, Peoples Republic of China.

${ }^{3}$ Edinburg-Tianjin Joint Research Centre for Systems Biology and Synthetic Biology, Tianjin University, Tianjin 300072, Peoples Republic of China. ${ }^{4}$ Key Laboratory of Systems Microbial Biotechnology, Tianjin Institute of Industrial Biotechnology, Chinese Academy of Sciences, Tianjin 300308, People s Republic of China. ${ }^{5}$ Department of Biochemical Engineering, School of Chemical Engineering and Technology, Tianjin University, Tianjin 300072, China.

Received: 22 September 2014 Accepted: 23 November 2014 Published online: 16 December 2014

\section{References}

1. Gao X, Chen JC, Wu Q, Chen GQ: Polyhydroxyalkanoates as a source of chemicals, polymers, and biofuels. Curr Opin Biotechnol 2011, 22:768 774

2. Choi Jl, Lee SY: High-level production of poly(3-hydroxybutyrate-co-3hydroxyvalerate) by fed-batch culture of recombinant Escherichia coli. Appl Environ Microbiol 1999, 65:4363 4368.

3. Choi J, Lee SY: Efficient and economical recovery of poly(3-hydroxybutyrate) from recombinant Escherichia coli by simple digestion with chemicals. Biotechnol Bioeng 1999, 62:546 553.

4. Lee SH, Kang KH, Kim EY, Chae TU, Oh YH, Hong SH, Song BK, Jegals J, Park SJ, Lee SY: Metabolic engineering of Escherichia coli for enhanced biosynthesis of poly(3-hydroxybutyrate) based on proteome analysis. Biotechnol Lett 2013, 35:1631 1637.

5. Kabir MM, Shimizu K: Gene expression patterns for metabolic pathway in pgi knockout Escherichia coli with and without phb genes based on RT-PCR. J Biotechnol 2003, 105:11 31.

6. Jung $Y M$, Lee JN, Shin HD, Lee YH: Role of tktA gene in pentose phosphate pathway on odd-ball biosynthesis of poly-beta-hydroxybutyrate in transformant Escherichia coli harboring phbCAB operon. J Biosci Bioeng 2004, 98:224 227.

7. Song BG, Kim TK, Jung YM, Lee YH: Modulation of talA gene in pentose phosphate pathway for overproduction of poly-beta-hydroxybutyrate in transformant Escherichia coli harboring phbCAB operon. J Biosci Bioeng 2006, 102:237 240

8. Lim SJ, Jung YM, Shin HD, Lee YH: Amplification of the NADPH-related genes zwf and gnd for the oddball biosynthesis of PHB in an E. coli transformant harboring a cloned phbCAB operon. J Biosci Bioeng 2002, 93:543 549.

9. Centeno-Leija S, Huerta-Beristain G, Giles-Gomez M, Bolivar F, Gosset G, Martinez A: Improving poly-3-hydroxybutyrate production in Escherichia coli by combining the increase in the NADPH pool and acetyl-CoA availability. Antonie Van Leeuwenhoek 2014, 105:687 696.

10. Postma PW, Lengeler JW, Jacobson GR: Phosphoenolpyruvate: carbohydrate phosphotransferase systems of bacteria. Microbiol Rev 1993, 57:543 594

11. Han MJ, Yoon SS, Lee SY: Proteome analysis of metabolically engineered Escherichia coli producing Poly(3-hydroxybutyrate). J Bacteriol 2001, 183:301 308.

12. Hong SH, Park SJ, Moon SY, Park JP, Lee SY: In silico prediction and validation of the importance of the Entner-Doudoroff pathway in poly(3-hydroxybutyrate) production by metabolically engineered Escherichia coli. Biotechnol Bioeng 2003, 83:854 863.

13. Su HS, Lang BF, Newman EB: L-serine degradation in Escherichia coli K-12: cloning and sequencing of the sdaA gene. J Bacteriol 1989, 171:5095 5102.

14. Zhang $X$, Newman E: Deficiency in L-serine deaminase results in abnormal growth and cell division of Escherichia coli K-12. Mol Microbiol 2008, 69:870 881.

15. Quail MA, Haydon DJ, Guest JR: The pdhR-aceEF-Ipd operon of Escherichia coli expresses the pyruvate dehydrogenase complex. Mol Microbiol 1994, 12:95 104.

16. Knappe J, Blaschkowski HP, Grobner P, Schmitt T: Pyruvate formate-lyase of Escherichia coli: the acetyl-enzyme intermediate. Eur J Biochem 1974, 50:253 263.

17. Netzer R, Peters-Wendisch P, Eggeling L, Sahm H: Cometabolism of a nongrowth substrate: L-serine utilization by Corynebacterium glutamicum. Appl Environ Microbiol 2004, 70:7148 7155.

18. Peters-Wendisch P, Netzer R, Eggeling L, Sahm H: 3-Phosphoglycerate dehydrogenase from Corynebacterium glutamicum: the C-terminal domain is not essential for activity but is required for inhibition by L-serine. Appl Microbiol Biotechnol 2002, 60:437 441.

19. Cicchillo RM, Baker MA, Schnitzer EJ, Newman EB, Krebs C, Booker SJ: Escherichia coli L-serine deaminase requires a [4Fe-4S] cluster in catalysis. J Biol Chem 2004, 279:32418 32425

20. Fu TF, Boja ES, Safo MK, Schirch V: Role of proline residues in the folding of serine hydroxymethyltransferase. J Biol Chem 2003, 278:31088 31094.

21. Miller BA, Newman EB: Control of serine transhydroxymethylase synthesis in Escherichia coli K12. Can J Microbiol 1974, 20:41 47.

22. Newman EB, Magasanik B: The Relation of Serine Glycine Metabolism to the Formation of Single-Carbon Units. Biochim Biophys Acta 1963, 78:437 448.

23. Lai $S$, Zhang Y, Liu S, Liang Y, Shang $X$, Chai X, Wen T: Metabolic engineering and flux analysis of Corynebacterium glutamicum for L-serine production. Sci China Life Sci 2012, 55:283 290.

24. Friehs K: Plasmid copy number and plasmid stability. Adv Biochem Eng Biotechnol 2004, 86:47 82.

25. Jones KL, Kim SW, Keasling JD: Low-copy plasmids can perform as well as or better than high-copy plasmids for metabolic engineering of bacteria. Metab Eng 2000, 2:328 338.

26. Yamane T: Yield of poly-D(-)-3-hydroxybutyrate from various carbon sources: a theoretical study. Biotechnol Bioeng 1993, 41:165 170.

27. Fraenkel DG, Levisohn SR: Glucose and gluconate metabolism in an Escherichia coli mutant lacking phosphoglucose isomerase. J Bacteriol 1967, 93:1571 1578.

28. Phue JN, Shiloach J: Transcription levels of key metabolic genes are the cause for different glucose utilization pathways in E. coli B (BL21) and $E$. coli K (JM109). J Biotechnol 2004, 109:21 30.

29. Abdel-Hamid AM, Attwood MM, Guest JR: Pyruvate oxidase contributes to the aerobic growth efficiency of Escherichia coli. Microbiology 2001, 147:1483 1498.

30. Nikel PI, Giordano AM, de Almeida A, Godoy MS, Pettinari MJ: Elimination of D-lactate synthesis increases poly(3-hydroxybutyrate) and ethanol synthesis from glycerol and affects cofactor distribution in recombinant Escherichia coli. Appl Environ Microbiol 2010, 76:7400 7406.

31. Nduko JM, Matsumoto K, Ooi T, Taguchi S: Effectiveness of xylose utilization for high yield production of lactate-enriched P(lactate-co-3hydroxybutyrate) using a lactate-overproducing strain of Escherichia coli and an evolved lactate-polymerizing enzyme. Metab Eng 2013, 15:159 166 
32. Le Meur S, Zinn M, Egli T, Thony-Meyer L, Ren Q: Poly(4-hydroxybutyrate) (P4HB) production in recombinant Escherichia coli: $\mathrm{P} 4 \mathrm{HB}$ synthesis is uncoupled with cell growth. Microb Cell Fact 2013, 12:123.

33. Quan J, Tian J: Circular polymerase extension cloning for highthroughput cloning of complex and combinatorial DNA libraries. Nat Protoc 2011, 6:242 251.

34. Spiekermann P, Rehm BH, Kalscheuer R, Baumeister D, Steinbuchel A: A sensitive, viable-colony staining method using Nile red for direct screening of bacteria that accumulate polyhydroxyalkanoic acids and other lipid storage compounds. Arch Microbiol 1999, 171:73 80.

35. Kuhlman TE, Cox EC: Site-specific chromosomal integration of large synthetic constructs. Nucleic Acids Res 2010, 38:e92.

36. Lin Z, Xu Z, Li Y, Wang Z, Chen T, Zhao X: Metabolic engineering of Escherichia coli for the production of riboflavin. Microbial Cell Factories 2014, 13:104.

37. Zhao J, Li Q, Sun T, Zhu X, Xu H, Tang J, Zhang X, Ma Y: Engineering central metabolic modules of Escherichia coli for improving beta-carotene production. Metab Eng 2013, 17:42 50.

38. Li ZJ, Cai L, Wu Q, Chen GQ: Overexpression of NAD kinase in recombinant Escherichia coli harboring the phbCAB operon improves poly(3-hydroxybutyrate) production. Appl Microbiol Biotechnol 2009, 83:939 947.

39. Zhu N, Xia H, Wang Z, Zhao X, Chen T: Engineering of acetate recycling and citrate synthase to improve aerobic succinate production in Corynebacterium glutamicum. PLoS One 2013, 8:e60659.

40. Boynton ZL, Bennett GN, Rudolph FB: Intracellular concentrations of coenzyme $A$ and its derivatives from Clostridium acetobutylicum ATCC 824 and their roles in enzyme regulation. Appl Environ Microbiol 1994, 60:39 44

41. Fu J, Wang Z, Chen T, Liu W, Shi T, Wang G, Tang YJ, Zhao X: NADH plays the vital role for chiral pure D-(-)-2,3-butanediol production in Bacillus subtilis under limited oxygen conditions. Biotechnol Bioeng 2014, 111(10):2126 2131.

42. Livak KJ, Schmittgen TD: Analysis of relative gene expression data using real-time quantitative PCR and the 2(-Delta Delta C(T)) Method. Methods 2001, 25:402 408.

doi:10.1186/s12934-014-0172-6

Cite this article as: Zhang et al.: Engineering of Serine-Deamination pathway, Entner-Doudoroff pathway and pyruvate dehydrogenase complex to improve poly(3-hydroxybutyrate) production in

Escherichia coli. Microbial Cell Factories 2014 13:172.

\section{Submit your next manuscript to BioMed Central and take full advantage of:}

$凶$ Convenient online submission

$\otimes$ Thorough peer review

$\nabla$ No space constraints or color $\nabla$ gure charges

$\otimes I m m e d i a t e$ publication on acceptance

Q Inclusion in PubMed, CAS, Scopus and Google Scholar

$\otimes$ Research which is freely available for redistribution 